\title{
Bistability conditions between lasing and non-lasing states for vertical-cavity surface-emitting lasers with frequency-selective optical feedback
}

\author{
A. Naumenko ${ }^{a}$, N.Loiko $^{a}$, K. Jentsch $^{b}$, T. Ackemann ${ }^{c}$ \\ ${ }^{a}$ Institute of Physics, NASB, 68 Nezalezhnasti Ave., 220072 Minsk, Belarus \\ ${ }^{b}$ Institute for Applied Physics, University of Muenster, \\ Corrensstr. 2-4, 48149 Muenster, Germany \\ ${ }^{c}$ SUPA and Department of Physics, University of Strathclyde, \\ 107 Rottenrow, Glasgow G4 ONG, Scotland, UK
}

\begin{abstract}
This paper gives analytical treatment and experimental details on on/off-bistability in vertical-cavity surfaceemitting lasers with frequency-selective feedback by a grating. In particular, the conditions for the coexistence of lasing and nonlasing states and an abrupt turn-on behaviour at threshold are derived using an envelope approximation. The theoretical and experimental results are in satisfactory agreement.
\end{abstract}

Keywords: semiconductor laser, VCSEL, frequency-selective feedback, bistability

Copyright 2007 Society of Photo-Optical Instrumentation Engineers.

This paper was published in Proc. SPIE. 6725, 672522, 2007 (in: Y. Kivshar, N. Rosanov (editors): Nonlinear Space-Time Dynamics) and is made available as an electronic reprint with permission of SPIE. One print or electronic copy may be made for personal use only. Systematic or multiple reproduction, distribution to multiple locations via electronic or other means, duplication of any material in this paper for a fee or for commercial purposes, or modification of the content of the paper are prohibited.

\section{INTRODUCTION}

This paper is devoted to investigations of the influence of delayed spectrally-filtered optical feedback on the operation characteristics of a vertical-cavity surface-emitting laser (VCSEL). In edge-emitting semiconductor lasers, external frequency-selective optical feedback along is known to provide stabilization of single-longitudinal mode operation ${ }^{1-4}$ but also to induce bi- or multi- stability between different emission states of the laser. ${ }^{5-9}$ In particular, in Ref. ${ }^{7}$ a filter-induced bistability between solitary laser- and external cavity- states was observed.

Bistability between lasing and non-lasing states and as a result an abrupt turn-on at threshold was found experimentally and theoretically in a recent paper for a VCSEL with optical feedback from a holographic diffraction grating. ${ }^{10}$ The bistability arises due to the interplay of the frequency selectivity of the grating, phase-amplitude coupling and thermal shifts of the emission frequency. In the present paper, we provide an analytical treatment of these phenomena and further experimental details.

This type of bistability could be useful for all-optical switching in photonic applications. In broad-area devices, the bistable characteristic might give rise spatial self-localized emission states (cavity solitons), ${ }^{11}$ which are considered as "bits" in future all-optical information processing schemes. ${ }^{12}$ Also, frequency-selective feedback provides additional possibilities for the design of laser schemes with higher controllability in comparison to conventional external optical feedback (e.g., for chaotic encryption applications), especially in conjunction with polarization degrees of freedom. 


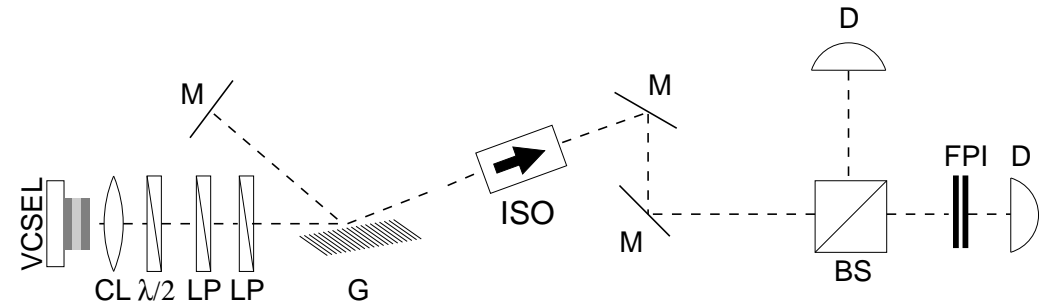

Figure 1. Experimental setup: CL collimating lens, $\lambda / 2$ half-wave retardation plate, LP linear polarizer, G grating, M HR-mirror, ISO optical isolator, BS beam splitter, D low-bandwidth detector, FPI scanning Fabry-Perot interferometer.

\section{EXPERIMENT}

The experimental setup is an amended version of the one described in $^{10}$ (Figure 1). The VCSELs investigated are gain-guided devices with an aperture diameter of $15 \mu \mathrm{m}$ from EMCORE Corp. (emission wavelength in the $850 \mathrm{~nm}$ region) operating in the fundamental transverse mode close to threshold (up to approx. $20 \%$ above threshold). The light is collected by an aspheric, anti-reflection coated lens. The external cavity is set up in Littman configuration, i.e., a holografic reflection grating (2000 lines/mm, Au coated) is hit at grazing incidence and the first diffraction order is retro-reflected by a plane highly-reflecting mirror. The bandwidth of the bandpass is estimated to be about $20 \mathrm{GHz}$ from the size of the light spot on the grating. The zeroth order is coupled out and used for detection. The length of the external cavity is about $31 \mathrm{~cm}$.

A combination of polarizing components is used to change the feedback strength. The final polarizer is adjusted to transmit the component orthogonal to the grooves of the grating. This is the component with the higher diffraction efficiency $(36 \%)$. This polarizer ensures a defined polarization state on the grating. A second polarizer is used to attenuate the feedback by turning it with respect to the final one. A half-wave plate is used to adapt the main principal axes of the VCSEL to the transmissive direction of the second polarizer. Since not all elements were anti-reflection coated, the losses were quite high and the maximum feedback level achievable in the setup is about $7 \%$. The maximally observed threshold reduction is $22 \%$, though $17-18 \%$ are more typically because alignment is very critical. This matches observations with feedback from a normal mirror that the maximally achieved feedback strength is about $70-80 \%$ of the theoretical value for a very carefully aligned setup ${ }^{13}$ but as low as about $40 \%$ otherwise. ${ }^{14,15}$

The laser is isolated from the detection path by an optical isolator with an isolation of more than $60 \mathrm{~dB}$. A lowbandwidth detector is used to monitor the time-averaged, polarization resolved light-current (LI-) characteristics. The polarization resolved optical spectrum is measured with a plano-planar scanning Fabry-Perot interferometer with a finesse of 200 and a free spectral range of $51 \mathrm{GHz}$.

The free-running laser has a threshold of about $6.36 \mathrm{~mA}$. The LI-curve is approximately linear and the turnon behavior is continuous (see the rightmost line in Figure 2a). This is a usual behavior of a laser, which shows a supercritical bifurcation from the non-lasing to the lasing state. One of the linear polarization components is strongly dominating the emission.

For the strongest achievable feedback (nominally 7\%) and a suitable detuning condition, there is an abrupt switch-on, after some shoulder. The appearance of the shoulder can be interpreted in two ways: First, the threshold behavior is supercritical and the laser emits on an external cavity mode with low intensity until this loses stability and the laser jumps onto the mode with high intensity. Another reason of the shoulder might be the occurrence of high-frequency oscillations before the abrupt switch-on which are averaged in time by our experimental detection system $\left(\mathrm{see}^{10}\right)$. (Numerical simulations indicate that the shoulder should be lower in height and more flat in this case, though.) If the current is increased further, this LI-curve shows a gradual decrease of output power, which is due to the fact that the laser gradually leaves the optimum operating point at the maximum of the filter due to the current induced red-shift $\left(\mathrm{see}^{10}\right)$. If the current is reduced again, the LI-curve is traced back approximately but extends beyond the up-switching point until there is again an abrupt switch-off. There is clear hysteresis and hence bistability. 
(a)

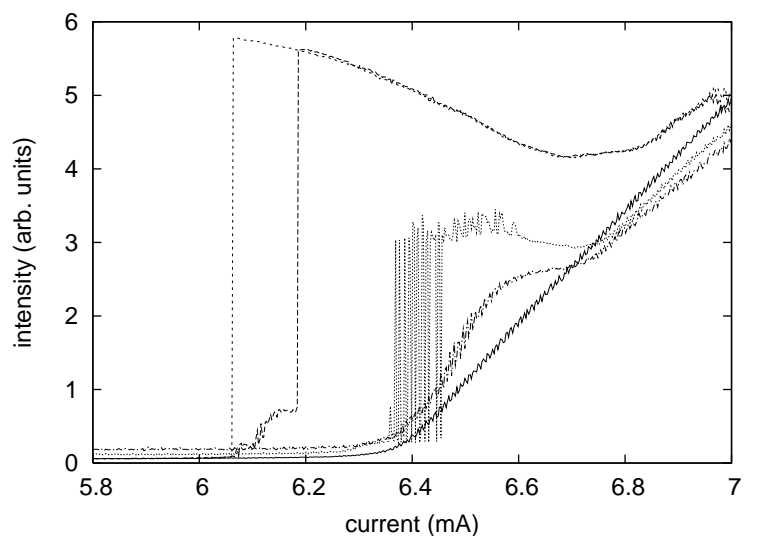

(b)

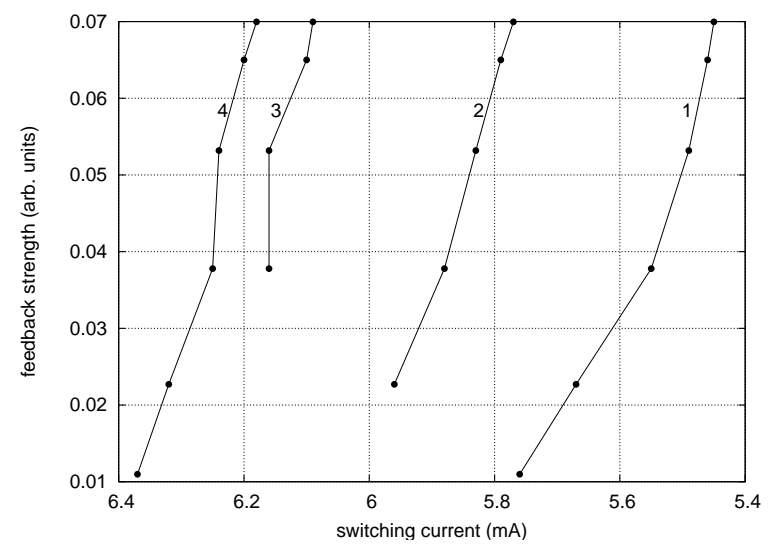

Figure 2. (a) Laser output power in dependence on injection current for different feedback level. From left to right: $7 \%$ (down- and up-scan), 1.1\% (up-scan), 0.04\% (up-scan), 0\% (up-scan). In the two latter cases up- and down scan are identical except for noise. The grating frequency is the same as for the left line of figure (b). (b) The feedback strength versus the pump current for abrupt switch-on events. Lines correspond to fixed values of the grating frequency.

If the feedback strength is reduced, the switching point moves to higher current, in tendency, and the amplitude of the switching decreases. For some feedback levels we have observed a "pure" abrupt switch-on (i.e. without a shoulder). At a feedback level of nominally 1.1\% (third to left curve in Figure 2a), there is still an abrupt switch-on but afterwards the laser does not stay on the high-amplitude state but there are irregular switching-events between a low-amplitude and a high-amplitude state. The details vary from scan to scan and the up- and down scans are also similar without a measurable hysteresis. This is interpreted as noise-induced back-and-forth switching from the grating-controlled high-amplitude state to the essentially solitary laser lowamplitude state and vice versa. It indicates that the 'potential barrier' between the two bistable states is low and thus one can anticipate that bistability will vanish soon, if the feedback strength is decreased further. Indeed, for a nominal feedback strength of $0.39 \%$ the switch-on of the laser is clearly supercritical (second to right curve of Figure 2a). The transition point from a supercritical switch-on to a subcritical one is found to be around a nominal feedback strength of $0.5 \%$.

The above mentioned dependence between the feedback strength and the current at which abrupt switch-on events occur is shown in Figure $2 \mathrm{~b}$ for four values of the detuning between the solitary laser frequency and the grating frequency. The feedback strength was changed by rotation of the leftmost polarizer along with the half-wave plate. A rotation to the left and to the right gave slightly different results probably due to a resulting misalignment and imperfect coupling. The corresponding difference reached about $0.03 \mathrm{~mA}$.

Figure 3a demonstrates a decrease of the height of the intensity jump with increasing switching current and - via Figure $2 \mathrm{~b}$ - feedback strength. Figure $3 \mathrm{~b}$ gives the dependence of the switching current versus the grating frequency for abrupt switch-on events at the maximal value of the feedback strength $(7 \%)$. The origin of the grating frequency is arbitrary. The straight line corresponds to the solitary laser dependency of the lasing frequency versus the pump current (only the slope has a meaning). The comparison of the two slopes implies that the effective detuning (i.e., the sum of the initial detuning and the current-induced red shift) at the switching point is constant across the measurement. A shoulder similar to the one discussed for the first LI-curve in Figure 2a is observed also for the upper points of Figure 3b, i.e. for high switching currents and a large initial detuning (low currents correspond to high detunings here).

Below we present our analytical treatment of these phenomena and results of numerical simulations. 
(a)

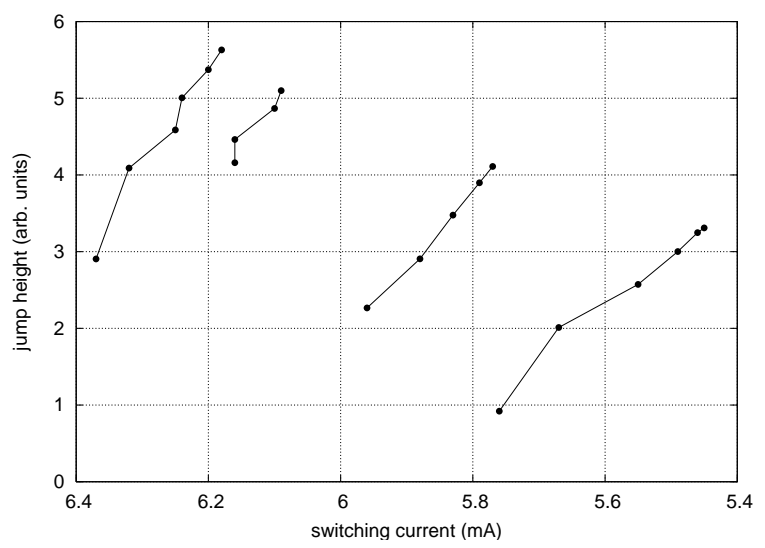

(b)

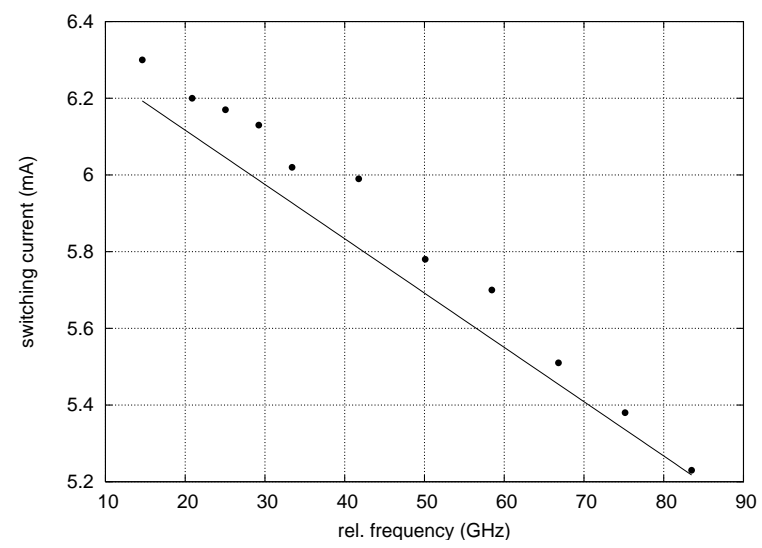

Figure 3. (a) Jump height versus the pump current for abrupt switch-on events. The values of the feedback strength and the grating frequency are the same as in the Figure 2. (b) The switching current versus the grating frequency for abrupt switch-on events at the maximal value of the feedback strength (7\%). The origin of the grating frequency is arbitrary. The straight line corresponds to the solitary laser dependency of the lasing frequency versus the pump current.

\section{MODEL EQUATIONS}

Polarization and spin dynamics of a VCSEL based on a quantum-well active medium can be described in the framework of the so called spin-flip-model (SFM). ${ }^{17}$ The modified rate equations describing the polarization dynamics in a VCSEL with frequency selective optical feedback from a distant diffraction grating have the following form ${ }^{10}$ :

$$
\begin{aligned}
\frac{d E_{ \pm}}{d t} & =\kappa(1+i \alpha)\left(N_{ \pm}-1\right) E_{ \pm}+i \Delta \omega_{0} E_{ \pm}-\gamma E_{\mp}+F_{ \pm}+W_{ \pm} \\
\frac{1}{\Gamma} \frac{d N_{ \pm}}{d t} & =\mu-N \mp \frac{\gamma_{s}}{\Gamma} D-2 N_{ \pm}\left|E_{ \pm}\right|^{2}-W_{N \pm}
\end{aligned}
$$

Here, $E_{-}$and $E_{+}$are the left and right circularly polarized components of the slowly varying amplitude of the electromagnetic field; $E_{\text {opt }}(t)=\operatorname{Re}\left(E_{ \pm}(t) \exp \left(i \omega_{0} t\right)\right)$, where $\omega_{0}$ is an arbitrary reference frequency. They are connected to the $\hat{x}$ - and $\hat{y}$ - linearly polarized components of the field $E_{x}$ and $E_{y}$ by $E_{ \pm}=\frac{1}{\sqrt{2}}\left(E_{x} \pm i E_{y}\right) . N$ is the total population difference between the conduction and valence bands, $D$ is the difference of the population differences for the two allowed transitions between the magnetic sublevels associated with right and left circularly polarized light. $N_{ \pm}=N \pm D$ are the population differences for the two allowed transitions. $\mu$ is the normalized injection current, which takes the value 1 at the solitary laser threshold (without anisotropies). $\gamma_{s}$ is the decay rate for the difference $D$ which is reduced by both spontaneous emission and spin-flip relaxation processes, $\Gamma$ is the decay rate of the total carrier population $N$ (we use a typical value of $1 / \Gamma \equiv \tau_{e}=1 \mathrm{~ns}$ ). $\gamma=\gamma_{a}+i \gamma_{p}$ denotes the anisotropies. $\gamma_{a}$ is the anisotropy of the field loss rate (positive $\gamma_{a}$ gives the $\hat{y}$ - polarized component a lower threshold). $\gamma_{p}$ represents the linear birefringence of the cavity (which gives opposite frequency shifts for the different linearly polarized fields of the solitary laser). $\kappa$ is the mean of the decay rates of the two linearly polarized components of the field; $\alpha$ is the linewidth enhancement factor. $\Delta \omega_{0}$ is the solitary laser frequency, which is measured - as all optical frequencies - with respect to the reference frequency $\omega_{0}$. Due to the shift of the cavity resonance because of Joule heating, the solitary laser frequency has the following phenomenological dependence on the injection current (see, e.g., ${ }^{8,9}$ ):

$$
\Delta \omega_{0}=\Delta \omega_{0}^{t h}-k_{\mu}(\mu-1)
$$


where $\Delta \omega_{0}^{t h}$ is the value of the frequency at the onset of lasing and the coefficient $k_{\mu}$ can be obtained from experiments. The effect of filtered feedback from a diffraction grating is calculated as:

$$
\bar{F}(t)=\frac{1}{2 \pi} \int_{-\infty}^{+\infty} d \omega \hat{K}(\omega) \int_{-\infty}^{+\infty} d t^{\prime} \bar{E}\left(t^{\prime}\right) \exp \left(i \omega\left(t-t^{\prime}\right)\right)
$$

where column vector $\bar{E} \equiv\left(E_{+}, E_{-}\right)$denotes the vectorial optical field, $\bar{F} \equiv\left(F_{+}, F_{-}\right)$and $\hat{K}(\omega)$ is the full matrix transfer function of the external cavity. Only one field round-trip in the external cavity is considered here. The polarization principal axes of the grating are aligned with the ones of the VCSEL, but the diffraction efficiency is anisotropic. The operation frequency of the laser with feedback will be denoted by $\Omega$. Hence, the transfer function can be represented as: $\hat{K}(\Omega)=\hat{\sigma} h(\Omega) \exp \left(-i \tau\left(\Omega+\omega_{0}\right)\right)$, where $\tau$ is the external cavity round-trip time. The diagonal elements of the matrix $\hat{\sigma}$ are equal to $\frac{1}{2}\left(\sigma_{x}+\sigma_{y}\right)$ and the nondiagonal ones are $\frac{1}{2}\left(\sigma_{x}-\sigma_{y}\right)$. Here, $\sigma_{x, y}$ represent the feedback strength for the x- and y- polarized components, where $\sigma_{x, y}=\left(1-r_{2}^{2}\right) r_{x, y} /\left(r_{2} \tau_{i n}\right)$. $r_{2}$ is the amplitude reflectivity of the outcoupling mirror of the VCSEL and $r_{x, y}$ are the corresponding values for the grating (on peak) for the two orthogonal polarization components, $\tau_{i n}$ is the VCSEL cavity round-trip time. $h(\Omega)$ is a normalized frequency dependent part of the transfer function. For the Littman configuration considered here, the light emitted by the VCSEL undergoes a double reflection from the diffraction grating before returning into the laser. Hence, the transfer function $h(\Omega)$ is given as the square of a normalized reflection coefficient of the grating:

$$
h(\Omega)=\left(\exp \left(-i T\left(\Omega-\omega_{m}\right)\right) \frac{\sin T\left(\Omega-\omega_{m}\right)}{T\left(\Omega-\omega_{m}\right)}\right)^{2} .
$$

Here, $\omega_{m}$ is the frequency of the main grating maximum. $1 / T$ is the grating bandwidth. It is related to the half width at half maximum, HWHM, of the double-reflection transfer function $h(\Omega)$, Eq. (4) by $T=x_{0} / \mathrm{HWHM}$, where $x_{0}$ is the root of the equation $\left(\sin x_{0} / x_{0}\right)^{4}=1 / 2$ and $x_{0} \simeq 1.0019$.

For the use in simulations, the above integral equation for the feedback term $\bar{F}(t)$ is reformulated as a system of differential equations with delay terms. Making use of the fact that the Green function describing the single reflection from the grating is $G(t)=\exp \left(i \omega_{m} t\right)[\operatorname{sign}(\mathrm{t})-\operatorname{sign}(\mathrm{t}-2 \mathrm{~T})] /(4 T)$ (see, e.g., $\left.{ }^{9}\right)$, the double reflection can be described by $\bar{F}_{j}(t)=\frac{1}{2 T} \int_{t-2 T}^{t} d t^{\prime} \bar{F}_{j-1}\left(t^{\prime}-\tau / 2\right) \exp \left(i \omega_{m}\left(t-t^{\prime}\right)\right)$, where the index $j$ on the lhs takes the values $j=\{1,2\}, \bar{F}_{0}(t) \equiv \bar{E}(t)$ and $\bar{F}(t)=\hat{\sigma} \exp \left(-i \omega_{0} \tau\right) \bar{F}_{2}(t)$ or in differential form ${ }^{10}$ :

$$
\frac{d}{d t} \bar{F}_{j}(t)=\frac{1}{2 T}\left[\bar{F}_{j-1}(t-\tau / 2)-\bar{F}_{j-1}(t-\tau / 2-2 T) \times \exp \left(i \omega_{m} 2 T\right)\right]+i \omega_{m} \bar{F}_{j}(t) .
$$

We take into account the Langevin noise sources $W_{ \pm}$and $W_{N \pm}$ that arise from spontaneous emission processes only, because it is not expected that the results depend on the details of the noise process. They have the following form ${ }^{18}: W_{ \pm}=\sqrt{\beta_{n} \kappa \Gamma N_{ \pm}} \xi_{ \pm}(t)$ and $W_{N \pm}=\frac{1}{2 \kappa}\left(W_{ \pm}^{*} E_{ \pm}+\right.$c.c. $)$, where $\beta_{n}$ is the spontaneous emission factor (the fraction of the spontaneously emitted photons that goes into the lasing modes); $\xi_{ \pm}$are two independent complex noise sources with zero mean and the correlation $\left\langle\xi_{ \pm}(t) \xi_{ \pm}^{*}\left(t^{\prime}\right)\right\rangle=2 \delta\left(t-t^{\prime}\right)$. In numerical simulations they are represented as $\xi_{ \pm}(t)=\chi_{ \pm} / \sqrt{\Delta t}$, where $\chi_{ \pm}$are complex Gaussian random variables (with zero mean and standard deviation $\left\langle\chi_{i} \chi_{i}^{*}\right\rangle=2$ ), and $\Delta t$ is the time interval over which the noise is held constant.

Due to the large uncertainty in the experimental parameters ( $\alpha$-factor, bandwidth of feedback, reflectivity of outcoupling mirror, intra-cavity losses and coupling efficiency to external cavity), a quantitative comparison between experiment and theory is quite difficult. The values $R_{2}=0.994$ and $\kappa=250 \mathrm{~ns}^{-1}$ (the latter corresponds to $R_{1}=0.998, \tau_{i n}=24 \mathrm{fs}$, index $n_{g r}=3.6$ and intracavity losses $a=20 \mathrm{~cm}^{-1}$ ) suggested in ${ }^{14}$ for VCSELs of the same type and $\alpha=5$ proved to be reasonable in our previous studies. ${ }^{10,15,16}$ The HWHM of the grating is $14.4 \pi \mathrm{GHz}$ for the double pass (in accordance with a single-pass bandwidth of $20 \mathrm{GHz}$ ).

The theoretically estimated maximal threshold reduction $(\Delta \mu=21 \%$ in the coupled cavity approach) for the highest feedback strength (nominally $7 \%$ or $\sigma_{x}=66 n s^{-1}$ ) is quite close to the one obtained experimentally ( $22 \%$ maximally and $17-18 \%$ more typically, see above). 
(a)

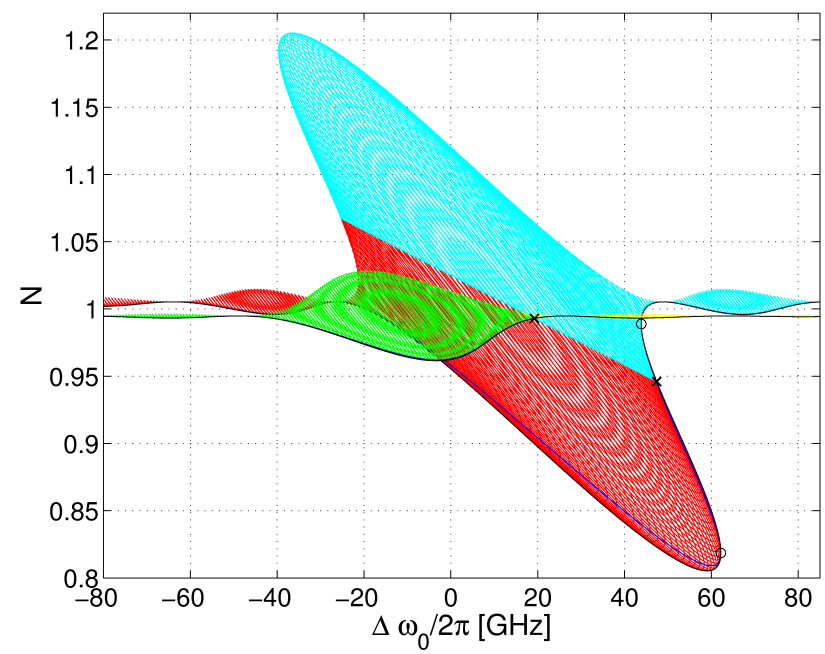

(b)

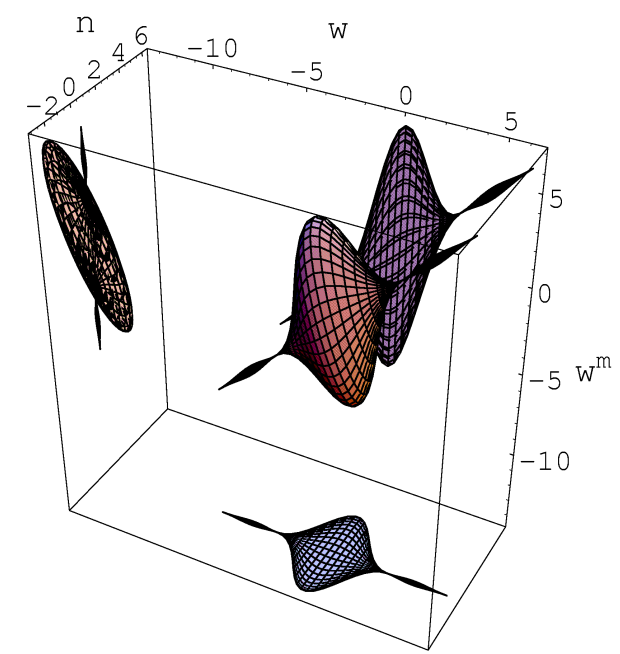

Figure 4. (a) Sets of the steady states in the domains $\left(\Delta \omega_{0}, N\right)$. The set with a high/low amplitude corresponds to x-/ylinearly polarized solutions. Dark grey lines denote lasing modes (above threshold). Black lines denote En $\mathbf{n}^{1}$ envelopes, open circles ("o") and crosses ("x") denote limit and threshold points in the envelope approximation. Thin grey line closed to the $\mathbf{E n}^{1}$ envelope is the image (map) of the $\mathbf{E w}^{1}$ - envelope onto the space. (b) The steady state surface given by eq. (8) in the domain $\left(w^{m}, w, n\right)$-space and its projections onto the corresponding two-dimensional subspaces.

\section{ANALYTICAL CONSIDERATIONS}

Steady states (monochromatic solutions) of the system (1), (3) in the absence of noise are found in the form $E_{ \pm}=Q_{ \pm} e^{ \pm i \psi+i \Omega t}$. For our experiments (Section 2), the locking of circularly polarized states to linearly polarized $\mathrm{x}$ - and y-steady-state solutions (or external cavity modes) is more typical due to the strong anisotropy (see, e.g., ${ }^{17}$ ). They were given in. ${ }^{10}$ A typical example is presented in Figures 4, where we display the space spanned by the frequency $\Delta \omega_{0}$ and the total population inversion $N$. Dark grey lines denote the location of modes satisfying the threshold condition $\mu \geq \mu_{t h}$ as given by equation (2). In accordance with that, the threshold line has the simple shape

$$
\Delta \omega_{0}=\Delta \omega_{0}^{t h}-k_{\mu}(N-1) .
$$

Note that we assume $\omega_{m}=0$, i.e. the reference frequency is the grating frequency.

To analyze the steady-state set, we introduce its envelope as the geometrical locations of the extremal (maximal/minimal) steady-state points in the space $\left(\Delta \omega_{0}, N\right)$. The black lines in figure 4 denote the $\left(\Delta \omega_{0}\right.$, $N$ )- lower envelope $\mathbf{E n}^{1}$. It is evident that the envelope $\mathbf{E n}^{1}$ in Figure 4a is S-shaped for x- linearly polarized steady-state solutions. The two limit points are denoted by open circles ("o"). Hence, in the area between the two limit points there is the possibility to observe the bistability between two different emission states with the same polarization. One is strongly affected by the feedback, whereas the other one corresponds essentially to the emission of the solitary laser. In between there is a third steady-state solution which is always unstable (the two others might be stable or unstable depending on parameters). y-polarized steady-states have a low amplitude and their low envelope does not display a bistability.

Crosses ("x") denote the threshold points where the laser switches on, if the current is increased from zero. For the nonlasing solution, increasing the current corresponds to a movement along the line $N=-\Delta \omega_{0} / k_{\mu}+1+$ $\Delta \omega_{0}^{t h} / k_{\mu}$ starting in the lower right corner (see eq. (6)). The threshold is encountered where this line intersects with the envelope of the set of steady-states. The slope is given by $-1 / k_{\mu}$ which is fixed for a specific device, but the threshold can be varied by changing the threshold solitary-laser frequency $\Delta \omega_{0}^{\text {th }}$, which corresponds to moving the line up and down. (This was done, for example, in the experimental measurements leading to 
Fig. 3b.) In particular, it can be arranged that the threshold point is between the two limit points. In that case, there is bistability between the feedback-effected state and the off-state between the threshold point and the right limit point. An abrupt (subcritical) turn-on of the laser to a high-amplitude emission is encountered because the threshold steady-state (cross) is unstable and the laser moves to the state on the lower envelope.

For convenience, we introduce the normalized value of the operating frequency $w=T\left(\Omega-\omega_{m}\right)$. Correspondingly, $w_{0}^{t h}=T\left(\Delta \omega_{0}^{t h}-\omega_{m}\right)$ denotes the normalized initial detuning between the (average) cavity resonance and the grating at the threshold of the solitary laser. Then the normalized detuning, $w^{m}$, for a linearly polarized state is given by $w^{m}=T\left[\Delta \omega_{0}-\omega_{m}-\left(-\alpha \gamma_{a}+\gamma_{p}\right)(-1)^{l}\right]$, where $l=0(1)$ corresponds to $\hat{x}^{-}\left(\hat{y}^{-}\right)$polarized states. Similarly, the normalized initial detuning, $\Delta$, for a linearly polarized state is $\Delta \equiv w_{0}^{t h}-T\left[-\alpha^{\prime} \gamma_{a}+\gamma_{p}\right](-1)^{l}=w^{m}+\beta m$ (here, $\beta \equiv k_{\mu} / \kappa$ and $\alpha^{\prime} \equiv \alpha-\beta$ ). The normalized values for the population inversion $(n)$ and for the injection current $(m)$ are $n \equiv T\left[\kappa(N-1)-(-1)^{l} \gamma_{a}\right]$ and $m \equiv T\left[\kappa(\mu-1)-(-1)^{l} \gamma_{a}\right]$. In addition, we use the following definitions: $\lambda \equiv T \sigma, \Lambda \equiv \sqrt{1+\alpha^{2}} \lambda$. Using this normalization, the steady states are given by:

$$
w-w^{m}=-\lambda \Pi(w)(\alpha \cos \phi-\sin \phi), \quad n=-\lambda \Pi(w) \cos \phi,
$$

where $\Pi(w)$ is the normalized absolute value of the filter reflectivity (the filter shape or the filter profile): $\Pi(w) \equiv\left|h\left(w / T+\omega_{m}\right)\right|$, and $\phi \equiv \operatorname{Arg}(\mathrm{h})-\tau\left(\Omega+\omega_{0}\right)$ is the full feedback phase. For the case of a double reflectivity from the grating considered here, the filter profile is $\Pi(w)=(\sin w / w)^{2}$ and the full feedback phase $\phi=-2 w-\tau\left(\Omega+\omega_{0}\right)$. $n)^{19}$ :

The steady states are situated on the ellipse-like curve in the space of the normalized inversion-frequency $(w$,

$$
n^{2}+\left[w-w^{m}-\alpha n\right]^{2}=\lambda^{2} \Pi^{2}(w)
$$

In the three-dimensional $\left(w^{m}, w, n\right)$-space, this equation describes a whirligig-like steady-state surface (see Figure 4b). (Equations (7) give this surface in the parametric form.) The envelopes of the solution (or mode) structure in the spaces $\left(w^{m}, w\right)$ and $\left(w^{m}, n\right)$ can be obtained from the corresponding extrema of the curve of steady-states (8). Geometrically, these envelopes can be envisaged as the extrema of the projections of the steady-state surface in the three-dimensional $\left(w^{m}, w, n\right)$-space onto corresponding two-dimensional subspaces (Figure 4b).

In accordance with that, the analytical expression for the envelope En (in the $\left(w^{m}, n\right)$-space) can be found by differentiation $d / d w$ from (8) and using $d w^{m} / d w=d n / d w=0$, which results in:

$$
w-w^{m}=\lambda \Pi(w)\left( \pm \alpha \sqrt{1-\lambda^{2} \Pi^{\prime 2}(w)}+\lambda \Pi^{\prime}(w)\right), \quad n= \pm \lambda \Pi(w) \sqrt{1-\lambda^{2} \Pi^{\prime 2}(w)} .
$$

The upper and the lower signs correspond to the upper $\left(\mathbf{E n}^{\mathbf{u}}\right)$ and the lower $\left(\mathbf{E n}^{\mathbf{l}}\right)$ boundaries of the mode structure in the space $\left(w^{m}, n\right)$. The value of the full feedback phase $\phi$ corresponding to the modes belonging to the En envelope is given by $\sin \phi^{n}=\lambda \Pi^{\prime}(w)$. Analogously, the envelope Ew in the space $\left(w^{m}, w\right)$ has the following form:

$$
w-w^{m}= \pm \Lambda \Pi(w), \quad n= \pm \Lambda \Pi(w) \alpha /\left(1+\alpha^{2}\right)
$$

The value of the full feedback phase $\phi$ corresponding to the modes belonging to the upper $\left(\mathbf{E w}^{\mathbf{u}}\right)$ and lower $\left(\mathbf{E w}^{\mathbf{l}}\right)$ envelope is the same as for the case of conventional feedback: $\phi_{ \pm}^{w}=(1 \pm 1) \frac{\pi}{2}-\varphi^{\alpha}$ correspondingly, where $\tan \varphi^{\alpha}=1 / \alpha$.

The limit points (or bistability boundaries) can be determined, e.g., from the envelope $\mathbf{E w}^{\mathbf{l}}$ (10) by using $d w^{m} / d w=0$, which results in:

$$
\Lambda \Pi^{\prime}(w)=-1
$$


The limit points lie on the blue-side of the filter maximum where $\Pi^{\prime}(w)<0$, if this derivative of the profile exists. Thus, the bistability domain is given by the inequality: $\Lambda \Pi^{\prime}(w) \leq-1$. To obtain the bistability boundaries $(\mathbf{B})$, eq. (11) has to be solved along with eq. (10). It is evident that the bistability domain does not depend on any parameter of the system in the normalized parameter space $\left(w^{m}, \Lambda\right)$ and is a function of the filter shape $\Pi(w)$ only. From the above considerations, we can obtain the bistability condition:

$$
\Lambda \geq 1 / y_{1}
$$

where $y_{1}=-\min \Pi^{\prime}(w)$. According to (12), the bistability can be found (in an appropriate interval of the initial detuning) only, if the normalized feedback strength is greater than a value $\Lambda_{\text {min }}=1 / y_{1}$. Reformulated for the original variables, the bistability condition (12) reads

$$
\mathcal{B} \equiv \frac{\sqrt{1+\alpha^{2}} \sigma}{2 H W H M} \geq \frac{1}{2 x_{0} y_{1}},
$$

where the variables on the right hand side are again only determined by the filter profile. The corresponding numerical value for the case of the Littman configuration is $1 /\left(2 y_{1} x_{0}\right)=0.924$ (with $\left.y_{1}=0.540\right)$.

Analogous expressions can be obtained for the case that multiple round trips of the field in the external cavity are taken into account. This can be important for high feedback levels. In particular, the equations for the steady state curve/surface can be represented in the following form:

$$
\left[\tilde{n}-r^{2} /\left(1-r^{2}\right)\right]^{2}+\left[\tilde{w}-\tilde{w}^{m}-\alpha \tilde{n}\right]^{2}=r^{2} /\left(1-r^{2}\right)^{2},
$$

where $r(\tilde{w})=r_{2}\left|r_{3}(\tilde{w})\right|$ and the values with the tilde are obtained from their values in the single round-trip approximation by $\tilde{x}=x / C$ with $C=\left(1-r_{2}^{2}\right) /\left(r_{2}^{2} \tau_{i n}\right)$. The envelopes, the bistability conditions, etc. can be found from this equation in the same way as discussed above.

The experimentally observed bistability-threshold feedback strength is about $0.5 \%\left(\sigma_{x}=17.7 n s^{-1}\right)$. This results in an bistability parameter (see eq. (13)) of $\mathcal{B}=1.005$, which is $9 \%$ greater than the corresponding theoretical value $\mathcal{B}=0.924$ for the threshold. Keeping in mind that some of the parameters are not well known (cf. the discussion above), this is regarded as a satisfactory agreement. In order to give an example for the sensitivity of the results on parameters, the choice of a higher value of the reflectivity $R_{2}=0.995$ (instead of $R_{2}=0.994$ used above) leads to the estimation for the bistability parameter $\mathcal{B}$ which is $10 \%$ smaller than the corresponding theoretical value for the bistability threshold.

Figure 5 presents the bistability domains (thick lines) in the space spanned by the normalized feedback strength $\Lambda$ and the normalized full detuning $w^{m}$ (left panel (a)), and in the space spanned by the renormalized pump current (using $\left.\kappa \Delta \mu \equiv \kappa(\mu-1)=\kappa\left(J-J_{t h}\right) / J_{t h} \simeq m / T\right)$ and the feedback strength $\sigma$ (right panel (b)). The two panels can be regarded to display related information, once in normalized units, once in the physical ones. The left (right) curves $\left(\mathbf{B}^{\mathbf{l}}\right.$ and $\mathbf{B}^{\mathbf{r}}$ ) in Figure 5a contain the left (right) limit points of the bistable envelopes (Figure 4). They correspond to situations were the jump of the intensity at the abrupt turn-on event is maximal (or zero). The shape of the bistability domain in the normalized parameter space $\left(w^{m}, \Lambda\right)$ does not depend on any parameter of the system and is a function of the filter shape $\Pi(w)$ only. According to the bistability condition (12), the bistability can be found (in an appropriate interval of the initial detuning) only, if the normalized feedback strength is greater than a value $\Lambda_{\min }=1 / y_{1}$. The bistability domains for different initial detunings $\Delta$ $(\Delta /(2 \pi T)=[-24.0,-9.5,6.0,11.2] G H z$ from right to left) are separated from each other in the physical space (Figure 5b).

In order to find the domain of bistability between the off-state and a grating-controlled state (denoted short as on/off-bistability domain in the following) we have to add the threshold conditions to the above considerations. They can be found in the envelope approximation using (9) for the lower envelope $\mathbf{E n}^{\mathbf{l}}$ (or the steady state surface, eq. (8) and the relations $m_{t h}=n$ and $\Delta=w^{m}+\beta m$. The corresponding threshold conditions and the threshold surface containing the threshold solutions are obtained from (9) and (8) simply by the following substitutions: $n \rightarrow m, w^{m} \rightarrow \Delta, \alpha \rightarrow \alpha^{\prime}$.

The set of nine threshold curves ( $\mathbf{T}$ ) located (on average) inside the V-shaped bistability domain is shown in Figure 5 a for different initial detunings $\Delta$ by thin lines marked as $1,2, \ldots, 9$. Analogously, the four threshold curves 
(a)

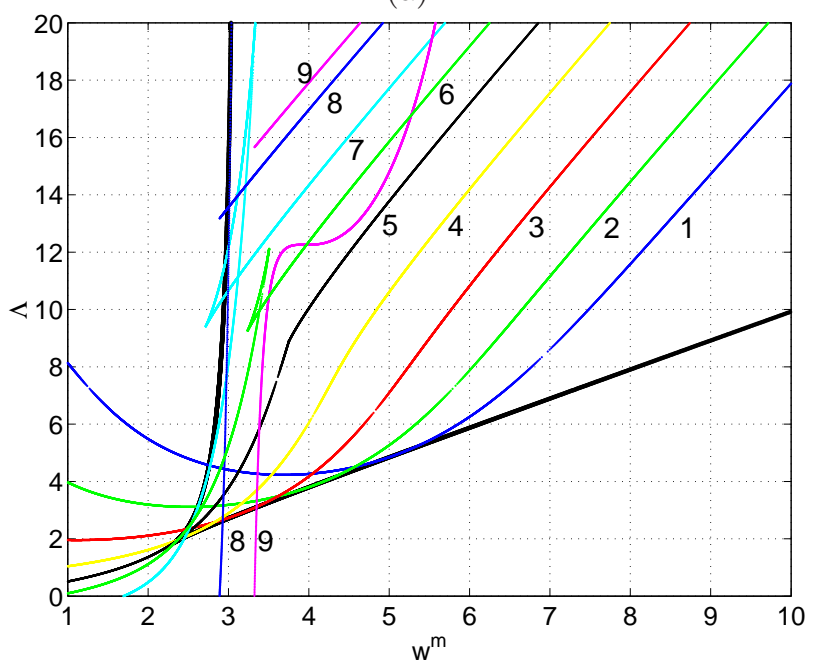

(b)

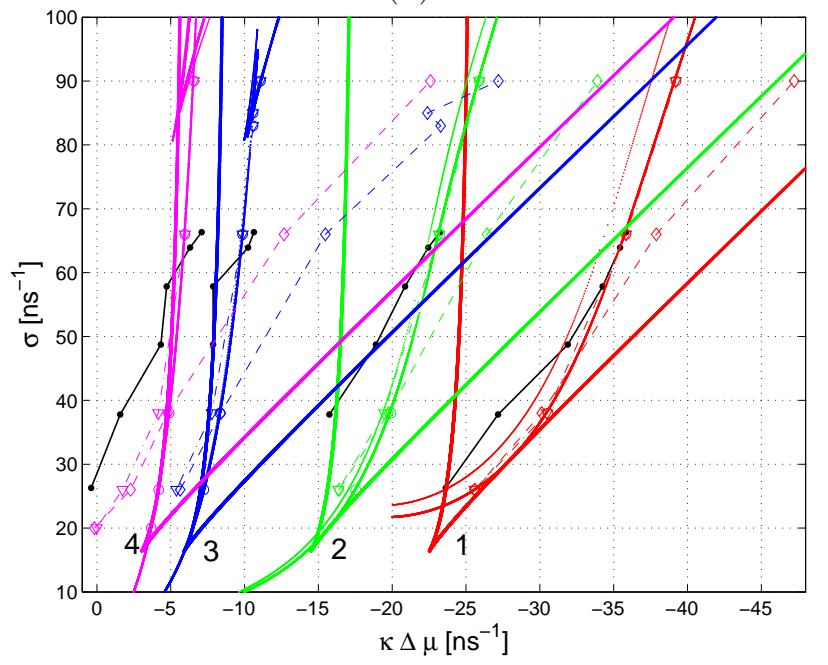

Figure 5. (a) Bistability domains in the space $\left(w^{m}, \Lambda\right)$ (thick line), and threshold curves (thin lines marked by $1,2, \ldots 9$ )

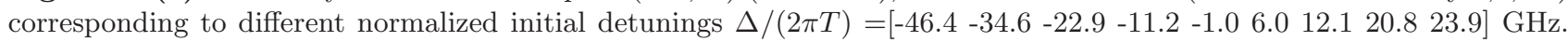
(b) Bistability domains in the space $(k \Delta \mu, \sigma)$ (thick lines) and threshold curves (thin lines) corresponding to different normalized initial detunings $\Delta /(2 \pi T)=[-24.0,-9.5,6.0,11.2] \mathrm{GHz}$ (from right to left). The thinnest lines (close to the threshold curves) give the threshold in multiple round trip approximation (14). The markers 'circle', 'triangle' and 'diamond' denote the numerically obtained laser threshold and the abrupt turn -on and -off events. The two latter ones are connected by dash-dotted and dashed lines correspondingly. Solid broken lines (marked by solid dots) are the experimental abrupt turn-on data shown in Figure 2b.

(T) for four different initial detunings $\Delta$ situated inside the four corresponding V-shaped bistability domains in the physical space of Figure $5 \mathrm{~b}$ are given by thin lines. In accordance with the analysis performed in, ${ }^{19}$ the contact points of the threshold curves with the bistability boundaries correspond to a transition between supercritical and subcritical (below or above the point, respectively) turn-on behavior. The on/off-bistability domain is situated between the right bistability boundary $\mathbf{B}^{\mathbf{r}}$ and the threshold curve $\mathbf{T}$ above (and to the right of) the point. In some interval of $\Delta$, the threshold curves contain a point of self-intersection creating a loop with the shape of a swallowtail (curves 5, 6, 7 of Figure 5a and curves 3, 4 of Figure 5b). For initial detunings, for which the self-intersection point is at a lower feedback strength than the contact point, this self-intersection point can be considered as being the exchange point between supercritical and subcritical turn-on behavior.

Geometrically, the formation of the considered swallowtail-like configuration corresponds to the situation when for the $\left(w^{m}, n\right)$-projection of the transparent 3D whirligig-like surface (8), the whirligig rim hides partially the whirligig axis (or, more exactly, starts to hide two diametrically opposite points of the whirligig axis, see Figure 4b). It will be accompanied by the corresponding metamorphoses of the En- envelope and therefore of the threshold curves $\mathbf{T}$ (see details $\mathrm{in}^{19}$ ). A swallowtail behavior can be observed only for the case $\lambda \geq 1 / z_{1}$, where $z_{1}=\max \sqrt{\frac{1}{2}\left(\Pi^{2}(w)\right)^{\prime \prime}}$. The corresponding threshold value for the "square-sinc" profile is $z_{1}=0.581$.

The thinnest lines (close to the threshold curves) in Figure 5b give the threshold determined in the multiple round-trip approximation (14). For the values of the feedback strength $\sigma$ presented in the figure, the difference between the two approaches is quite small, the larger for smaller initial detuning $\Delta$. In this figure, we give also for comparison the values of the pump current at which the abrupt switch-on was observed experimentally (solid broken lines marked by solid dots already shown in Figure 2b). One can see a similar tendency in the theory and in the experiment. In order to understand some of the differences, we have carried out numerical simulations. The results of these are presented below. 
(a)
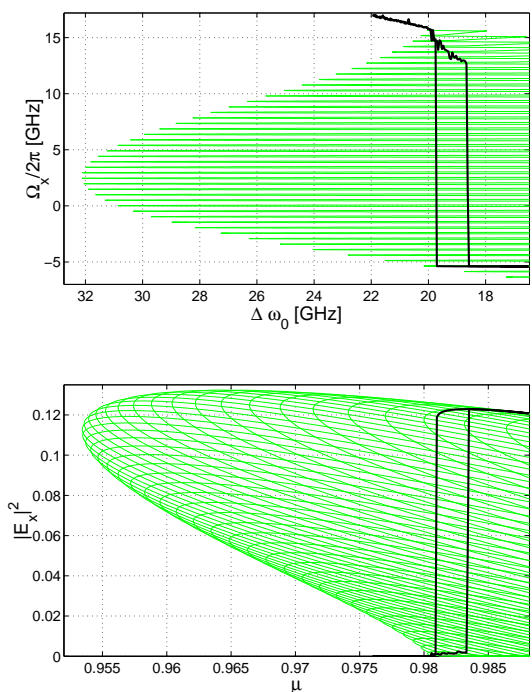

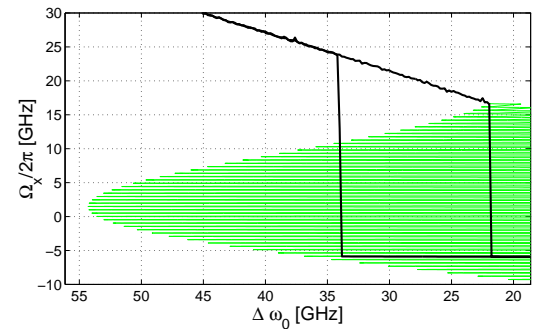

(b)

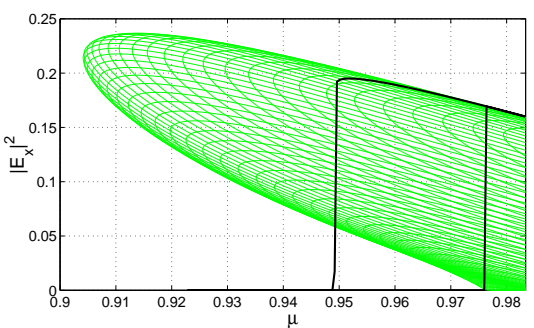

(c)
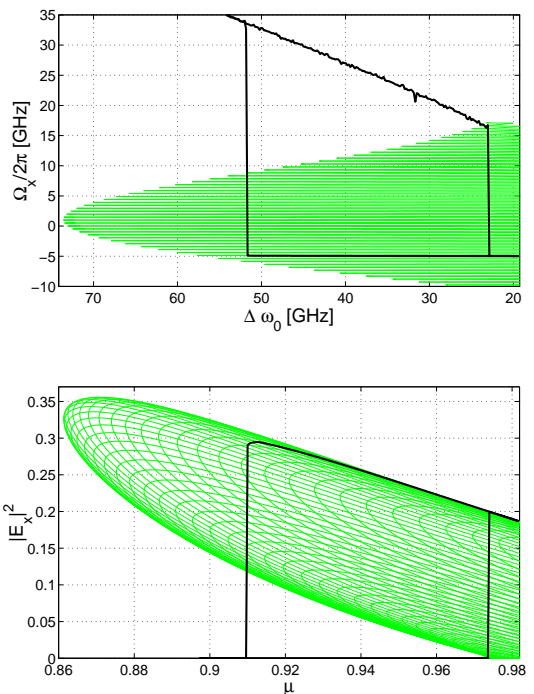

Figure 6. Evolution of the averaged intensities $\left(<\left|E_{x}\right|>^{2}\right)$ and of the averaged frequencies $\left(<\Omega_{x}>\right)$ for $E_{x^{-}}$polarized components (averaging time is $200 \mathrm{~ns}$ with sampling step $0.08 \mathrm{~ns}$ ) versus injection current (lower panels) or the frequency $\Delta \omega_{0}$ (upper panels). The thick solid curves denote the results obtained from numerical simulations for the $x$ - component. The thin lines denote the analytically obtained steady-state solutions. $\Delta /(2 \pi T)=11.2 \mathrm{GHz} \sigma \sigma=38 n s^{-1}$ (a), $66 n s^{-1}$ (b), $90 \mathrm{~ns}^{-1}$ (c).

\section{RESULTS OF NUMERICAL SIMULATIONS AND COMPARISON WITH EXPERIMENTS}

In order to integrate the model equations numerically, a forth-order variable-step method has been used. For low values of the feedback strength - i.e., $\sigma$ being smaller the bistability threshold (13)-, a smooth LI-curve with the supercritical turn-on usual for lasers is observed. This corresponds to the third experimental curve shown in Figure 2a. For a higher feedback strength (above the bistability threshold), the results depend on the value of the initial detuning $\Delta$. For rather large positive $\Delta$, the smooth supercritical turn-on is followed by an abrupt jump of the laser emission to a high level of the intensity. This behavior is typical for the bistability between lasing states of the solitary laser and feedback affected states. ${ }^{8,9}$

For a small values of the detuning, hysteresis between the off-state and the highest gain external-cavity mode can be observed. ${ }^{10}$ Typical examples of LI-curves for this case are presented in Figure 6. For comparatively small feedback strength, the LI-curves show a small pedestal before the abrupt turn-on (Figure 6a). The pedestal is the remnant of high-amplitude oscillations after time averaging. The pump current corresponding to the abrupt jump of the intensity is shifted to a higher value with respect to the threshold point. (We can call it a "shift due to the existence of intermediate attractive dynamical states" or shortly a "dynamical shift".) The hysteresis loop (with increasing/decreasing the current) for this case is quite narrow. For higher feedback levels, the pedestal disappears and an abrupt turn-on takes place exactly at the laser threshold (Figure 6b). Increasing the feedback strength leads to an enlargement of the hysteresis loop (Figure 6c). This was also observed in the experiment (Fig. 2). The effect is connected to the fact that at higher feedback levels the frequency (see the upper panels of Figure 6) of the most stable maximal-gain-mode (envelope En, containing the modes with the maximal gain) moves away from the edge (envelope $\mathbf{E w}$, containing the minimal frequency modes) well into the external cavity mode structure in $\left(\Delta \omega_{0}, \Omega\right)$-space, or $\left(w^{m}, w\right)$ - space respectively, for increasing feedback strength. At the backward scan of the injection current, the system tries to move along a previously selected external cavity mode up to the point where this mode disappears due to a saddle-node bifurcation. This corresponds to the point of abrupt turn-off point. We caution that the switch-off can occur slightly earlier depending on noise level and 
noise realization. As the images in the upper panels of Figure 6 illustrate, states with an intermediate frequency (close to the grating frequency) have a larger existence interval in current than the states close to the minimal frequency. Hence, the stability interval of the maximal gain mode - and therefore the width of the hysteresis loop - increases for increasing feedback strength. Analogous regularities were observed for the case of hysteresis between the lasing solitary laser state and the feedback affected state. ${ }^{8,9}$

The points corresponding to the laser thresholds, to the abrupt turn -on and -off events obtained in numerical simulations are denotes by 'circles', 'triangles' and 'diamonds' in Figure 5b. The latter (turn -on/off events) are connected to each other by dash-dotted/dashed lines for convenience. We see that the numerically calculated thresholds ('circles') lie exactly on the analytically obtained threshold curves (thin lines). For low feedback strength, the points of abrupt turn -on/off ('triangles' / 'diamonds') almost coincide with each other and are shifted quite strongly to the left of the threshold points due to the existence of the intermediate high-amplitude oscillations described above. For a larger initial detuning $\Delta$ (into the positive direction) this dynamical shift becomes larger. For moderate feedback level $\left(\sigma \sim 40 n s^{-1}\right)$, both the hysteresis loop between the on-off events and the value of the dynamical shift are small. At even higher feedback levels, the turn-on points ('triangles') coincide almost exactly with the threshold points (zero dynamical shift). The turn-off points ('diamonds') are strongly shifted to the right resulting in a large hysteresis loop. Again, a larger initial detuning $\Delta$ (into the positive direction) results in a widening of the hysteresis loop. (The sharp bend of the dashed curve 3 (with $\Delta /(2 \pi T)=6.0 \mathrm{GHz})$ marked by 'diamonds' at strong feedback level $\left(\sigma=83-85 n s^{-1}\right)$ are explained by the abrupt turn-on selects the 11th and 10th modes respectively (the numeration starts from the reddish mode.) Correspondingly, the hysteresis loop in second case are smaller.

Solid broken lines (marked by dots) (Figure 5b) are the experimentally observed abrupt turn-on data presented earlier in Figure 2b. Here, we superimpose them to the theoretical ones for a qualitative comparison. The qualitative behavior is quite similar, though the slopes of the theoretically and experimentally found turn-on curves are slightly different. In the theory, the slopes are determined approximately only by the value of $\alpha$ (and $\alpha^{\prime}$ ) at least for quite high filtering feedback levels: The higher $\alpha$ the smaller the slope. The observed discrepancy hints either to some mismatch of the parameters entering the calculation or to an uncertainty in the experimental determination of the threshold values. As already mentioned above, the results for opposite signs of rotation of the linear polarizer controlling the feedback strength differed somewhat indicating a parasitic misalignment.

In addition, an inspection of the LI-curves corresponding to the leftmost experimental curve in Figure $2 \mathrm{~b}$ shows the presence of a smooth supercritical switch-on followed by a subsequent abrupt turn-on for strong feedback levels. This is in contrast to the behavior at moderate feedback levels where at the same grating frequency a subcritical turn-on is observed (not shown). This behavior is in contradiction to the theory where for a fixed initial detuning only subcritical turn-on events are expected above a threshold value of the feedback strength.

Alternatively, the feedback-induced change of losses or the resulting shift of the threshold current can lead to a change of the effective temperature of the active medium (due radiation-cooling under lasing conditions) and therefore to a change of the effective "solitary" laser frequency $\Delta \omega_{0}$ (better to say cavity resonance condition). Hence, the latter can be a function of feedback parameters in a more sophisticated microscopic theory. Theoretical investigations on the influence of both the polarization and the angle misalignment can be useful to understand the inevitably non-ideal experimental situation. In particular, a more realistic shape of the filter transfer-function might explain some distinctive details observed in the experiment.

Nevertheless, we observe 5\% coincidence for the maximal threshold reduction in the theory and in the experiment, at least $9 \%$ coincidence for the bistability threshold and approximately $30 \%$ coincidence for the slope of the bifurcation (switch-on) lines in the Figure 5b. Hence, the totality of the observations indicate that the coincidence of the experimental and theoretical findings is not only qualitatively but also quite acceptable on a quantitative level.

It would be tempting to interpret the fractures (sharp bends or sharp changes in slope) of the two left experimental lines in Figure $2 \mathrm{~b}$ at high feedback levels as being indications for the swallow-tail fractures of the theoretical threshold curves $(\mathbf{T})$ arising at the same values of the initial detuning due to the swallow-tail catastrophe (see curves 6 and 7 in Figure 5a or corresponding to them curves 3 and 4 in Figure 5b). However, 
the swallow-tail bends appear in the theory only at feedback levels $30 \%$ higher than the experimental ones. A reduction of the bifurcation parameter $\lambda=\sigma T$ by about $30 \%$ can be achieved either by increasing $\sigma$ (e.g., by a reduction of $R_{2}$ up to the value 0.9915$)$ or by decreasing the $H W H M$ of the filter to $10 \mathrm{GHz}$. In order to keep the previous value of $\mathcal{B}$, the parameter $\alpha$ has to be decreased to 3 . However, this change of parameters would result in a strong decrease of conformity between the slopes for the theoretical and experimental turn-on curves and is considered hence as being unlikely. Probably, the irregular changes in slope of the experimental curves are due to misalignments arising if the feedback strength is changed (as discussed already above). We remark that the detection of the swallow-tail bends in the experimental turn-on curves would be interesting because it could be considered as confirmation of the correctness of the chosen shape for the grating transfer-function. I.e., the swallow-tail catastrophe can not be found at reasonable feedback levels in the case of the Lorenzian filter profile in contrast to the sinc- or square-sinc profile. Analogously, a relative comparison of the experimentally measured values of the initial detuning corresponding to the turn-on curves in Figure $2 \mathrm{~b}$ with the theoretical ones shows that these values can be quite good fitted for the case of square-sinc filter profile but not for the case of a Lorenzian one.

\section{CONCLUSION}

In this paper we compare the experimentally and theoretically found regularities for the on/off- bistable behavior of a VCSEL with external filtered optical feedback from a distant diffraction grating. The theoretical description of the phenomenon is based both on an analytical treatment, which is given within framework of the so-called envelope approximation, and on the direct numerical simulation. The comparison shows quite good qualitative agreement of the abrupt turn-on/off events with increasing/decreasing the injection current, of the bistability domains, of the parametric dependencies for the turn-on/off in the theory and in the experiment. In particular, the experimentally observed bistability threshold coincides with the theoretically predicted one to within $9 \%$. A less good accuracy $(\sim 30 \%)$ is obtained for the slopes of the abrupt turn-on curves in the space spanned by injection current versus the feedback strength. The analysis yields a quite acceptable quantitative coincidence between theory and experiment. Due to rather large uncertainties in the laser parameters and some drawbacks inherent both in the experiment and in the theory, a more exact comparison is difficult and a better coincidence probably can not be expected.

\section{Acknowledgements}

The experimental work in Münster was supported by the Deutsche Forschungsgemeinschaft. The initial phase of the collaboration was also supported by travel grants of the Deutsche Forschungsgemeinschaft. The present work of TA is supported by the EU project FunFACS.

\section{REFERENCES}

1. F. Favre and D. Le Guen, IEEE J. of Quantum Electron., vol. QE-21, pp. 1937-1946 (1985).

2. R. Wyatt and W.J. Delvin, Electron Lett., vol. 19, pp.110-112 (1983).

3. E. Brinkmeyer, W. Brennecke, M. Zurn, and R. Ulrich, Electron. Lett., vol. 22, pp. 134-135 (1986).

4. M. Kozuma, M. Kourogi, and M. Ohtsu, Appl. Phys. Lett., vol. 61, pp. 1895-1897 (1992).

5. P. Zorabedian, W.R. Trutna, and L.S. Cutler, IEEE J. of Quantum Electronics, vol. QE-23, pp. 1855-1860 (1987).

6. Chi Yang, X. Wang, and J.G. McInerney, IEEE J. of Quantum Electronics, vol. QE-232, pp. 813-821 (1996).

7. M. Giudici, L. Giuggioli, C. Green, and J.R. Tredicce, Chaos, Solitons and Fractals, vol. 9, pp. 1-8 (1999).

8. A.P.A. Fischer, O.K. Andersen, M. Yousefi, S. Stolte and D. Lenstra, IEEE J. of Quantum Electronics, vol. QE-36, pp. 375-384 (2000).

9. A. Naumenko, P. Besnard, N. Loiko, G. Ughetto and J.C. Bertreux, IEEE J. of Quantum Electron, vol. 39, pp. 1216-1228 (2003).

10. A. Naumenko, N. Loiko, M. Sondermann, K. Jentsch, T. Ackemann, Optics Commun. 259, 823-833 (2006). 
11. M. Sondermann, F. Marino, K. F. Jentsch, T. Ackemann, and R. Jäger. In Nonlinear Guided Waves and Their Applications, Toronto, March 28-31 (2004) Paper MB5; Y. Tanguy, T. Ackemann, W. J. Firth, R. Jäger, unpublished.

12. S. Barland, J.R. Tredicce, M. Brambilla, L.A. Lugiato, S. Balle, M. Giudici, T. Maggipinto, L. Spinelli, G. Tissoni, T. Knodel, M. Miller, R. Jager, Nature, vol. 419, pp. 699-702 (2002).

13. K.F.A. Jentsch, Charakterisierung von oberflächenemittierenden Halbleiterlasern mit optischer Rückkoppling. Diplomarbeit, Westfälische Wilhelms-Universität Münster (2006).

14. P.A. Judge, C.H.L. Quay, J.A. Hudgings, Appl. Phys. Lett. 81, 3933-3935 (2002).

15. A.V. Naumenko, N.A. Loiko, M. Sondermann, T. Ackemann, Phys. Rev. A 68, 033805 (2003).

16. T. Ackemann, M. Sondermann, A. V. Naumenko, and N. A. Loiko. Appl. Phys. B 77, 739-746 (2003).

17. M. San Miguel, Q. Feng and J. V.Moloney, Phys. Rev. A 52, 1728-1739 (1995).

18. J. Mulet, C.R. Mirasso, and M. San Miguel, Phys. Rev. A 64, 023817-1-8 (2001).

19. A. Naumenko, N. Loiko, T. Ackemann, submitted to Phys. Rev. A. 